\title{
Injection Device for Percutaneous Osteoplasty
}

\author{
Julien Garnon ${ }^{1,2}$, Laurence Meylheuc ${ }^{1}$, Léo Harrer ${ }^{1}$, Guillaume Koch ${ }^{2}$, Afshin \\ Gangi $^{2}$, Bernard Bayle ${ }^{1}$ \\ ${ }^{1}$ UMR 7357 CNRS, ICube - University of Strasbourg, 1 place de l'Hôpital, 67091 Strasbourg, \\ France \\ ${ }^{2}$ Department of Interventional Radiology, Nouvel Hôpital Civil, 1 place de l'Hôpital, 67096 \\ Strasbourg Cedex, France
}

\begin{abstract}
The purpose of this work is to present one major technical problem associated with extraspinal cementoplasty, and to present one potential future solution using a dedicated robot. Tests on cadaver bones with a robotic assistant dedicated to vertebroplasty, which requires low volumes of cement unlike extraspinal cementoplasty, are presented. Experimental and numerical mechanical tests are also presented showing the need to adapt the assistant to larger volumes.
\end{abstract}

\section{Introduction}

The injection of Polymethyl methacrylate (PMMA) into a vertebra using a percutaneous approach has been described for the first time in 1987 [1]. Since then, vertebroplasty has been applied to treat painful osteoporotic and malignant compression fractures of the spine. This technique provides excellent antalgic and functional outcomes for the patients, and is still performed worldwide to alleviate the pain related to spinal compression fractures. The use of cementoplasty outside the spine, also referred as extra-spinal cementoplasty or osteoplasty, has emerged in the meantime as a minimally invasive treatment option to manage osteolytic malignant tumours. For lesions located in weight baring areas (such as the acetabulum), osteoplasty pursues a double objective of pain palliation and bone consolidation [2]. The key feature of a cementoplasty procedure is to fill the osteolytic areas and the surrounding cancellous bone, while mitigating the risk of cement leakage that is the most common complication of such procedure. Hence, injection under real-time image guidance (with fluoroscopy and/or CT-scan) is mandatory to assess proper repartition of the PMMA bone cement. This exposes the intervening physician to a significant amount of radiation, as the injection is done manually close to the source of X-rays. Another current limitation in extra-spinal cementoplasty is the volume of cement: the vast majority of the PMMA kits on the market do not exceed $10 \mathrm{ml}$ in quantity. If this quantity is appropriate for 
an injection within a vertebral body, it is insufficient to provide consolidation in many extra-spinal cases. The required volume in areas such as the acetabulum or the long bones can range from 10 to $50 \mathrm{ml}$ depending on the extension of the lytic process. Modification of the technique of injection is therefore necessary.

Different strategies may be used to achieve an injection greater than $10 \mathrm{ml}$. The solution most commonly reported in the literature is to insert an additional needle inside the lesion after the previous injection has been completed, in order to add some more cement. However, this increases the number of punctures, the time of the procedure and may lead to a on cohesive interface between the different PMMA cement balls. Another solution is to deliver sequentially several volumes of cement through a single needle. As soon as the injection of a PMMA kit come to its end, another volume is prepared and subsequently injected within the same bone trocar. This allows to reduce the number of needle, theoretically increases the chance of having one large cohesive PMMA ball, but is technically demanding. The intervening physician has to deal with both the hardening of the first cement and the countdown for preparation of the next cement, thereby leaving little place for mistakes during preparation and connection. The purpose of the present work is to present our experience of sequential injection of cement using a dedicated robotic assistance designed for vertebroplasty, and discuss the potential evolution for a future version adapted for extra-spinal cementoplasty.

\section{Material and methods}

This study was conducted in the anatomy lab of the University Hospital of Strasbourg. The goal was to perform extra-spinal injection of cement using the robotic assistance initially designed for vertebroplasty, and to further study the bony reinforcement with mechanical and finite element analysis.

\section{Robotic bone cement injection setup}

An orthopedic cement injection device has been designed and produced in our laboratory $[3,4]$. This device allows to push cement into a cavity while keeping the practitioner away from X-rays. It is made up of a master device remotely controlled by a practitioner and a slave device located close to the patient (Fig. 1). The slave device allows injecting PMMA into bones during its polymerization while controlling its temperature in order to slow down the chemical reaction and therefore the hardening of the cement. The slave device consists of a syringe holder, a force sensor and an actuated linear carriage connected to the syringe plunger that pushes the cement through the needle into the bone. A motor located at the rear of the device allows to deliver a compression force level rising up to $2000 \mathrm{~N}$. A passive exchanger, composed of a sheath and eutectic gel, keeps the cement at low temperature and thus significantly increases the possible injection time range. 


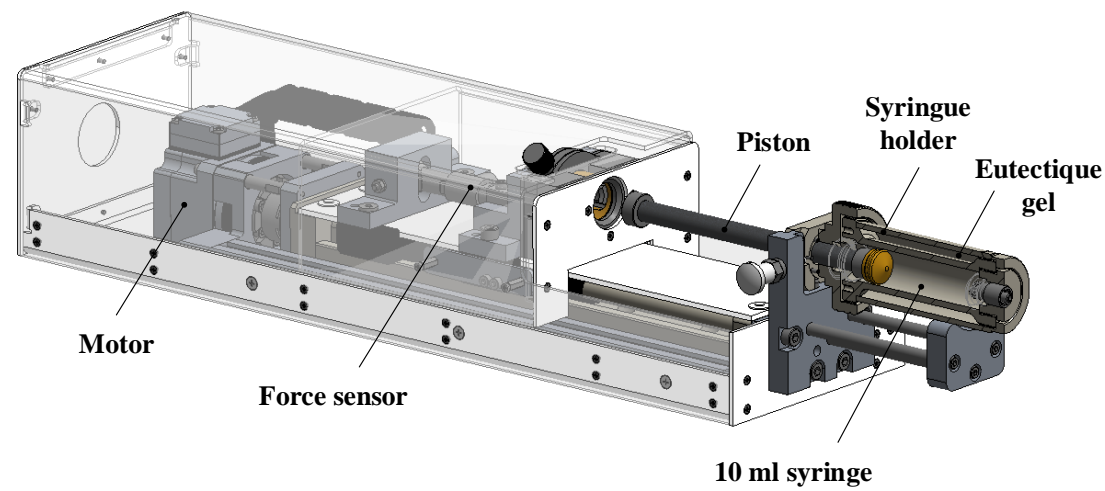

Fig. 1: CAD view of the slave device.

In vitro tests have shown that the time could be prolonged up to 30 minutes, for cement that usually sets within 10 minutes [5]. Theoretically, such device would be very well adapted for extraspinal cementoplasties, as it increases the time for injection and gives more time to the operator to properly fill the lesion through one bone trocar, while mitigating potentially the risks of leakages.

\section{Anatomic samples}

Two human humerus originating from two different cadavers were used for the present study. The long bones are not the most common site of injection in extra-spinal cementoplasty as cement presents little resistance to bending and torsion loads. However, these bones were selected as they were available in our institution, and the cancellous bone of the diaphysis is both easily approached with a single needle and compatible with the injection of more than $10 \mathrm{ml}$ of cement. Moreover, cement repartition can be quickly checked with stand-alone fluoroscopy, while it might be much more complex to monitor the injection in area with a complex shape such as the pelvic bone.

\section{Robotized bone cement injection in anatomic samples}

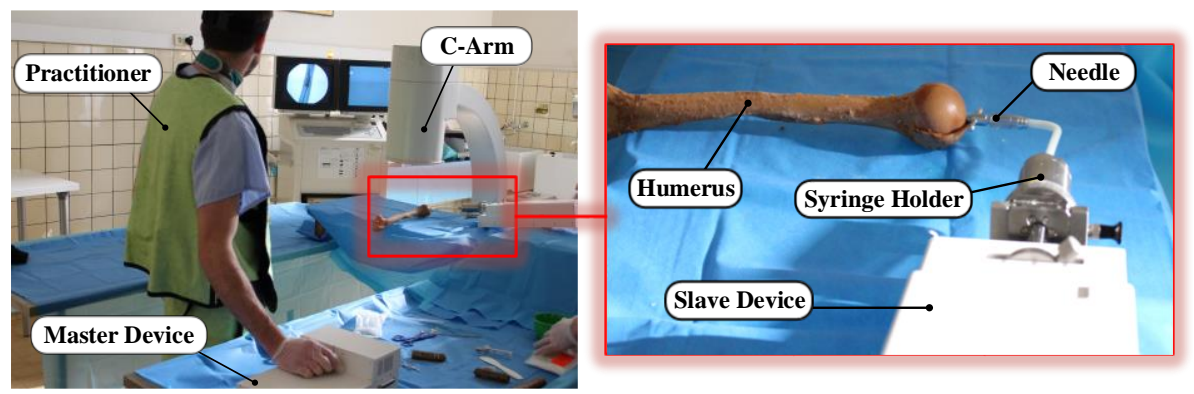

Fig. 2: Humerus cementoplasty with the assistive device.

A needle was inserted into the humeral head at the proximal epiphysis, as described in Fig. 2 and 3. The cement kits of PMMA and the syringe holder were kept 
cold before use $\left(10^{\circ} \mathrm{C}\right)$. The injection of the first volume of cement $(10 \mathrm{ml})$ was performed under intermittent fluoroscopic monitoring. When the injection was completed, another cement kit inserted in another syringe holder was immediately connected to the slave device and injection was pursued after the needle has been withdrawn from approximatively $5 \mathrm{~cm}$. The maneuver was repeated until the medullar cavity was completely filled, of if injection by the device had to be stopped due to cement hardening. In the later case, another needle was introduced in the bone and the same protocol was conducted. The final goal was to fill the cancellous bone entirely from proximal to distal.

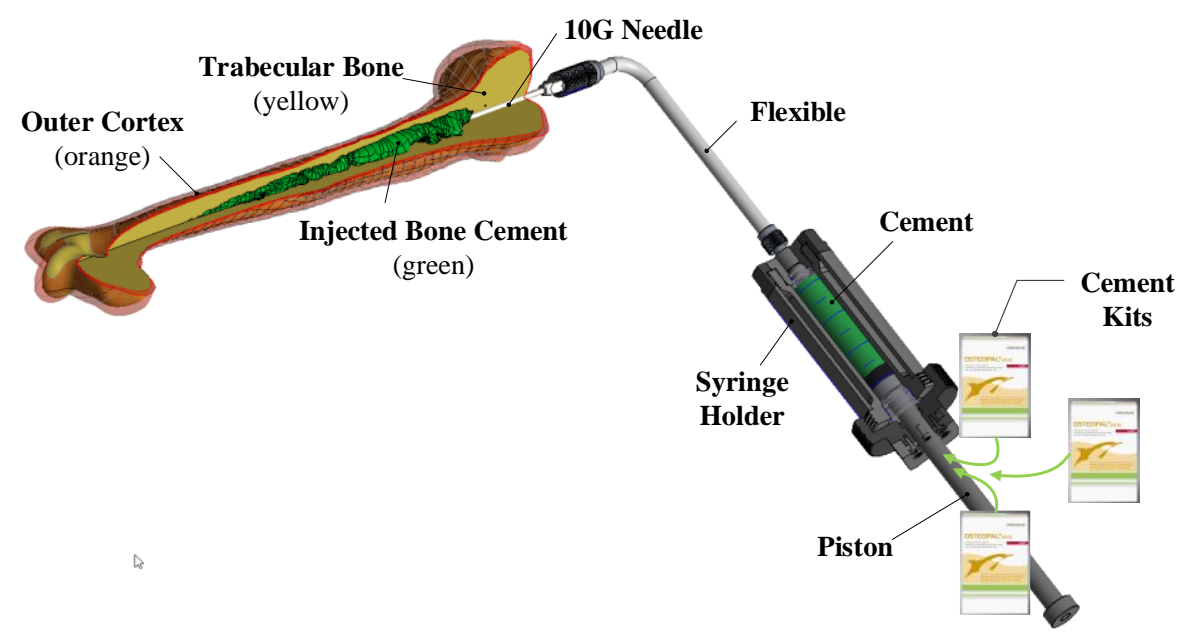

Fig. 3: Injection of cement kits.

\section{Imaging of the cement-augmented humerus}

Once the injection of the medullar cavity of both bones was completed, the needles were withdrawn. The two humerus were imaged with CT-scan, in order to detect material weld surfaces between two different cement kits and allow for further finite elements analysis. The result of an injection is presented in Fig. 3 and 4 on the reconstructed 3D representation of the bone cement for one of the two humerus.

\section{Mechanical Study}

Bending Tests - In order to measure the stiffness of the injected bones a three-point bending test was performed with a universal testing machine from Zwick. The bones were loaded with a constant crosshead speed of $1 \mathrm{~mm} / \mathrm{min}$. The tests were conducted until failure with specimens which were injected 3 weeks before testing. 


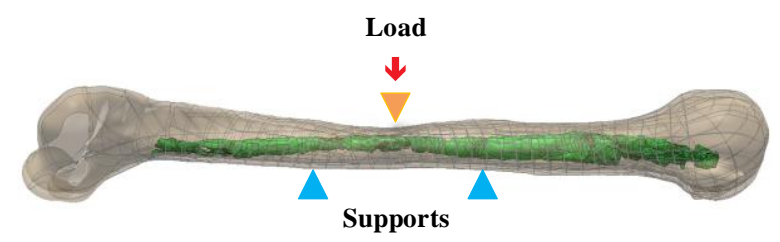

Fig. 4: Three points bending test.

FEM Analyses - A FEM model was setup to analyze the cemented bone behavior under the same three-point bending experimental conditions. The solid geometry of the injected bones, required to simulate the bone behavior after cementoplasty, was obtained by making 3D bone reconstruction based on the CT data. The joint use of a software dedicated to segment structures in 3D medical images and a CAD software made it possible to a numerical model the injected bones with the cement. Three solid components were segmented and reconstructed (Fig. 3), one representing the outer cortex, one representing the trabecular bone and the last representing the injected bone cement.

The mechanical characteristics of bones required for FEM analyses are difficult to determine for several reasons. Among those, the structure of bones is inhomogeneous and anisotropic. Then, it changes from a patient to another. Lastly, it changes with age. Several publications deal with the measurement of Young modulus by non-destructive experiments, followed by a homogenization [6,7]. So, in the light of these works, linear elastic and isotropic material properties were assumed for the outer cortex and the trabecular spongy bone (see Tab.1). The modulus of elasticity of the bone cement (Osteopal from Heraeus) were defined in [8].

\begin{tabular}{|l|l|l|l|}
\hline Material & Young Modulus & Poisson's ratio & Volume \\
\hline Cement (PMMA) & $2000 \mathrm{MPa}$ & 0.4 & $6.24 \mathrm{~cm}^{3}$ \\
\hline Outer Cortex & $16000 \mathrm{MPa}$ & 0.3 & $30 \mathrm{~cm}^{3}$ \\
\hline Trabecular Bone & $80 \mathrm{MPa}$ & 0.3 & $206 \mathrm{~cm}^{3}$ \\
\hline
\end{tabular}

Tab. 1: Material properties.

Supports and gradual loads were applied from 500 to 3000 N. The analyses were performed using Inspire 2019.1 software.

\section{Results}

\section{Anatomy Experimental Testing}

On a cadaver study performed on 2 humerus with imaging (CT-scan) correlations, we were able to demonstrate that the robotic-assisted injection allowed us to inject as 
much as $40 \mathrm{ml}$ without multiple punctures, thanks to the preparation of several syringes of cement that were injected one after the other on the same needle. We have found that, as in the in vitro tests, the sheath filling of eutectic gel, allows the cement to be kept cold long enough to ensure an injection without hardening of the cement in the syringe. However, this was associated with multiple manipulations of the syringe holder, due to the different kits used to reach $40 \mathrm{ml}$ of filling volume, and was not technically optimal. Hence, in one case the bone trocar had to be exchanged for another one after injection $20 \mathrm{ml}$ because of cement deposition within the cannula. This led to the lack of coalescence between the different cement streams, as we show in the following.

\section{Experimental and numerical analysis of bending tests}

Subsequent 3 points bending tests were carried out until the failure of the injected humerus. The rupture was initiated inside the injected bone, and then has grown to the cortical bone, as the load increased. Figure 5 shows a CT image of the two broken bones.

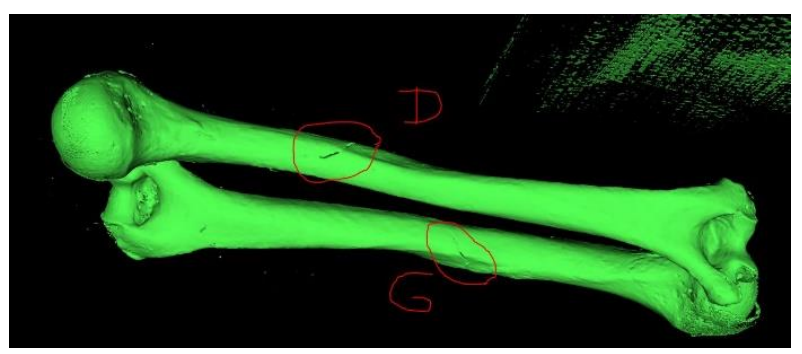

Fig. 5: The injected bones after bending tests.

The test machine sensor detected a first crack suggesting that the injected cement broke when the load was $3000 \mathrm{~N}$. In order to well understand what occurred in the treated bones, we choice to simulate the same 3 points bending test of a humerus (the right) with the material properties provided Tab. 1.

As we can see on the figure 6, the force-displacement curves obtained in the case of the experimental test and in the case of numerical study, are very close. These result show that the material properties and the $3 \mathrm{D}$ reconstruction of the humerus structure is satisfactory. These result show that the material properties and the $3 \mathrm{D}$ reconstruction of the humerus correlate, thereby supporting the efficacy of the developed FEM. 


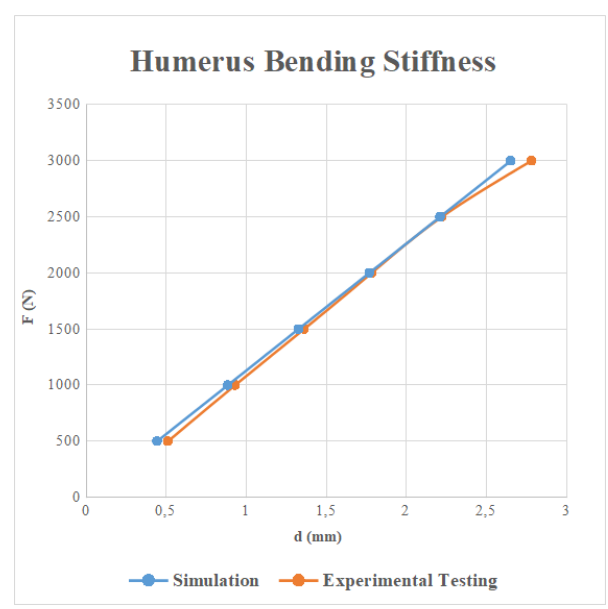

Fig. 6: Simulation and experimental bending tests.

The figure 7 shown the von Mises stress on the cortical and trabecular bone (Fig. 7 left) and on the injected cement (Fig. 7 right) for $3000 \mathrm{~N}$ of bending load. In order to better see the stress distribution, the displayed area has been limited around the supports and load.
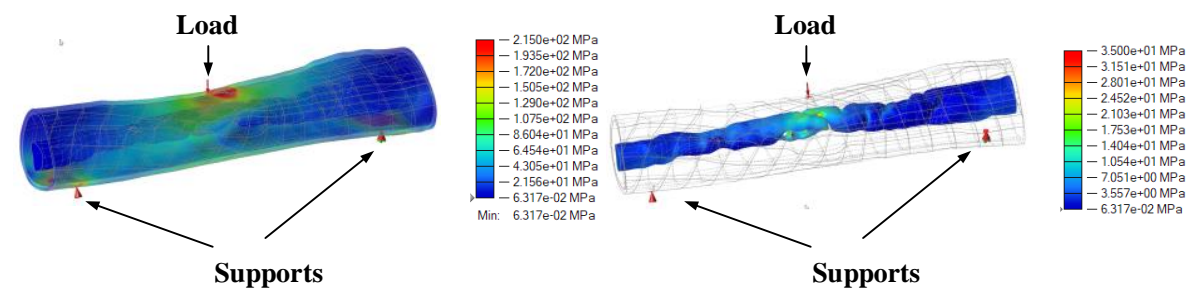

Fig. 7: Von Mises Stress for $3000 \mathrm{~N}$ of bending load.

We can notice that the maximum von Mises stress is located as we expected next to the load location but more precisely at the junction of the two cement kits. At the bending load of $3000 \mathrm{~N}$, the maximum von Mises stress of PMMA cement is $35 \mathrm{MPa}$. The ultimate tensile strength for the PMMA cement testing is approximatively 50-60 $\mathrm{MPa}$ [8]. The fact that the injected cement breaks at a lower value demonstrated that the fracture occurred in front of the welding surfaces of the two dements kits, suggesting the importance of having one single cohesive cement. 


\section{Discussion}

We demonstrated that the robot initially designed for vertebroplasty could be used to perform extra-spinal injection (in long bones in the present cases). We did not experienced a situation of cement hardening during the different injections, which is a common situation in the clinical practice using manual injection. However, the manipulation of the syringe holders during the exchange, in order to handle the injection of volume greater than $10 \mathrm{ml}$, was associated with the need of needle exchange during the procedure. Moreover, the mechanical tests outlined that failure might occur at the interface between different cement volumes, most likely due to non-cohesion between the successive PMMA kits. Impending fractures requiring stabilization with cement might therefore benefit from the injection of a single large $(>10 \mathrm{ml})$ volume of cement, which is hardly achievable with manual injection due to the rapid onset of polymerization (10-15 minutes). Hence, injection of a large volume of cement might benefit from the device with a passive exchanger used to cool down the PMMA. Ideally, the device has to be adapted to be compatible with larger syringes $(30 \mathrm{ml}$ would be adapted to the real clinical practice). This adaptation comes how-ever with specific issues that need to be addressed such as the pressure of injection, the nature and thickness of the insulation material and the design of the syringe that might impact the shear rate of cement and therefore its viscosity.

Another advantage of the device is the possibility for the operator to per-form the injection distant from the source of X-ray, which is associated with a de-crease of the scattered radiation for the interventional radiologist. This is of utmost importance especially for cases where large amount of cement is injected, and for which continuous fluoroscopic monitoring is performed for several minutes.

\section{Conclusion}

Extra-spinal cementoplasty of volumes of cement greater than $10 \mathrm{ml}$ using an injecion device designed for spine is feasible, but technically demanding due to the need for manipulations during syringe exchanges. Development of an injection device adapted for the delivery of larger volume of cement (up to $30 \mathrm{ml}$ ) would theoreticaly allow to inject one single cohesive PMMA ball while offering a prolonged time for injection that is mandatory to carefully check progressive filling of the lesion and avoid cement leakage.

\section{References}

[1] P. Galibert, H. Deramond, P. Rosat, and D. Le Gars, "[Preliminary note on the treatment of vertebral angioma by percutaneous acrylic vertebroplasty]," Neurochirurgie, vol. 33, no. 2, pp. 166 $168,1987$. 
[2] Z. Wang et al., "CT fluoroscopy-guided percutaneous osteoplasty for the treatment of osteolytic lung cancer bone metastases to the spine and pelvis," J Vasc Interv Radiol, vol. 23, no. 9, pp 1135-1142, Sep. 2012, doi: 10.1016/j.jvir.2012.06.007.

[3] N. Lepoutre, G. I. Bara, L. Meylheuc, F. Schmitt, L. Barbé, and B. Bayle, "Design and control of a thermal device for bone cement injection," in 2016 6th IEEE International Conference on Biomedical Robotics and Biomechatronics (BioRob), 2016, pp. 508-513, doi: 10.1109/BIOROB.2016.7523677.

[4] N. Lepoutre et al., "Robotically assisted injection of orthopedic cement: System design, control and modeling," in 2016 European Control Conference (ECC), 2016, pp. 2127-2132, doi: 10.1109/ECC.2016.7810606

[5] N. Lepoutre, L. Meylheuc, G. I. Bara, L. Barbé, and B. Bayle, "Bone cement modeling for percutaneous vertebroplasty," J. Biomed. Mater. Res. Part B Appl. Biomater., vol. 107, no. 5, pp. 1504 1515, Jul. 2019, doi: 10.1002/jbm.b.34242.

[6] D. T. Zaharie and A. T. M. Phillips, "Pelvic Construct Prediction of Trabecular and Cortical Bone Structural Architecture," J Biomech Eng, vol. 140, no. 9, 01 2018, doi: 10.1115/1.4039894.

[7] C. H. Turner, J. Rho, Y. Takano, T. Y. Tsui, and G. M. Pharr, "The elastic properties of trabecular and cortical bone tissues are similar: results from two microscopic measurement techniques," J Biomech, vol. 32, no. 4, pp. 437-441, Apr. 1999, doi: 10.1016/s0021-9290(98)00177-8.

[8] K.-D. Kühn, PMMA cements. Berlin: Springer Medizin, 2014. 